\title{
Permanent Magnet Couplings: Field and Torque three-Dimensional Expressions Based on the Coulombian Model
}

\author{
R. Ravaud, G. Lemarquand, V. Lemarquand and C. Depollier
}

\begin{abstract}
This paper presents three-dimensional expressions for the optimization of permanent-magnet couplings. First, we give a fully analytical expression of the azimuthal field created by one arc-shaped permanent magnet radially polarized which takes into account its magnetic pole volume density. Such an expression has a very low computational cost and is exact for all points in space. Then, we propose two semianalytical expressions of the azimuthal force and the torque exerted between two arc-shaped permanent magnets. These expressions are valid for thick or thin arc-shaped permanent magnets. Furthermore, this approach allows us to realize easily parametric studies and optimizations. The analytical approach taken in this paper, based on the Coulombian model, is a good alternative compared to the finite element method generally used to study such configurations.
\end{abstract}

\section{Index Terms}

Analytical, couplings, permanent magnet, azimuthal force, radial magnetization, torque

\section{INTRODUCTION}

A RC-SHAPED permanent magnets are widely used for the design of magnetic bearings or couplings. These devices have first been studied by Yonnet [1][2][3] and other authors have studied their properties as Elies [4][5] or Delamare [6]. Indeed, they allow to transmit a torque without mechanical contact and thus to have efficient devices. Baran [7] have showed that media used for manufacturing permanent magnets allow to have more and more efficient couplings. Furthermore, Samanta et al [8], Mukhopadhyay et al [9] and Moser at al [10] have proposed structures using permanent magnets allowing good performances.

As a consequence, the determination of magnetic fields and magnetic forces is of great interest in such structures. However, their expressions are often difficult to obtain analytically and alternative semianalytical expressions can be used. For instance, Furlani has proposed semi-analytical expressions of the magnetic field components created by polarized cylinders [11][12][13]. Moreover, Azzerboni et al [14][15][16] have proposed a three dimensional calculation of the magnetic field created by CurrentCarrying Massive Disks. This calculation is based on both elliptic integrals and numerical integrals which can be solved by using the Hermite polynomial decomposition.

Furthermore, geometrical methods have been proposed by Abele and Leupold [17] to calculate high uniform magnetic field sources [18] using wedge-shaped magnets- with sections presenting wedges or angles lower than $90^{\circ}$ - and extended from the Halbach magical structure [19][20][21]. These geometrical methods are useful because they are simple to use.

Manuscript Received September 26, 2008. Corresponding author: G. Lemarquand (e-mail: guy.lemarquand@ieee.org).

The authors are with the Laboratoire d'Acoustique de l'Universite du Maine UMR CNRS 6613, Avenue Olivier Messiaen, 72085 Le Mans Cedex 9, France, 
Babic et al use both Legendre's polynoms and Heumann functions to calculate the magnetic field components created by thin or thick arc-shaped permanent magnets at any point in space (either singular or regular) [22][23]. In addition, Selvaggi [24][25] uses a multiple representation of the source to calculate analytically the external magnetic field created by permanent magnets with Green's functions. Such an approach is interesting because it has a very low computational cost.

Morevoer, Kwon et al [26] have studied the effects of geometric asymmetries in magnet design. It is to be noted that generally, it is interesting to precisely know such effects because they can lower the quality of devices using permanent magnets.

Some methods whose goal is to obtain the magnetic field as quickly as possible have also been proposed. For example, Rakotoarison has proposed semi-analytical expressions based on only one numerical integration so as to determine the three magnetic field components created by arc-shaped permanent magnets whose polarization is radial [27]. Zhilichev [28] has studied the magnetic field created by two infinite cylindrical permanent magnets by using bipolar coordinates.

Another approach, proposed by Perigo [29], deals with the calculation of magnetic flux density produced by axially magnetized toroidal permanent magnets. At last, some approaches based on elliptical expressions have also been proposed by Durand [30] and by the authors [31]. Such expressions are useful since the algorithms used to determine elliptic integrals are very robust and very fast.

It can be noted that for several years, authors dealt with a two-dimensional approach to study the magnetic field created by arc-shaped permanent magnets [32]. The main reason is that a two-dimensional approach is fully analytical and allows an easy parametric optimization of the permanent magnet dimensions so as to obtain a great force in synchronous couplings [33][34][35]. However, this 2D-approach is not valid when we determine the magnetic field far from one magnet.

The determination of the force between permanent magnets has been proposed by Akoun and Yonnet [36] and is generally employed to study the magnetic forces exerted between ring permanent magnets. Other methods, using a three-dimensional approach have been discussed [37][38][39]. Such approaches give a precise value of the force exerted between curved permanent magnets. These approaches differ from the one used in this paper because the expressions determined by Conway are based on Bessel function integrals. The main interest of his approach lies in the fact that expressions based on Bessel integrals are simple to use and the numerical evaluation of such special integrals is fast and precise. The approach proposed by Kim is also interesting: its method is close to the one established in this paper. He has obtained numerical expressions based on elliptic integrals but has used the vector potential whereas we use neither the scalar potential nor the vector potential for calculating the magnetic field created by an arc-shaped permanent magnet.

The calculation of the force between two ring permanent magnets whose polarization is axial has been also proposed [40][30].

All the semi-analytical or analytical approaches taken by the authors allow to optimize devices using permanent magnets [41][42]. Moreover, they allow to compare these devices in order to have the best compromise between its effectiveness [43][44] and its cost. It is to be noted that the properties of permanent magnets have been improved [45]. This allows to build original structures which are very efficient [46][47][48][49]. Moreover, permanent magnets can be used to design original devices involved in the micromass measurement [50].

This paper has three main objectives. In the first part, we present a fully analytical expression of the azimuthal field created by one arc-shaped permanent magnet whose polarization is radial. A similar expression had been published in a previous paper [31] but we had neglected the magnetic pole volume density. In this paper, the expression obtained is valid for thin or thick arc-shaped permanent magnets. Thus, we can easily determine the demagnetizing field with such an expression. 
The second aim of this paper is to present a semi-analytical expression of the azimuthal force exerted between two arc-shaped permanent magnets whose polarization is radial. The main difficulty lies in the fact that this calculation requires up to six integrations to determine this azimuthal force. We show in this paper that we can reduce these six integrations and the semi-analytical expression proposed is very simple to use. We also discuss the possibility of neglecting some terms in the expression obtained so as to have a very low computational cost.

Eventually, the third aim of this paper is to propose a semi-analytical expression of the torque transmitted between two arc-shaped permanent magnets. The calculation of the torque between two arc-shaped permanent magnets radially magnetized is also difficult because the magnetic pole volume densities of each magnet must be taken into account. However, we show in this paper that we can determine this torque precisely and quickly. Such an expression is in fact very useful because it allows precisely optimizing the arc-shaped permanent magnet dimensions so as to obtain the greatest torque.

The semi-analytical expressions of the azimuthal force and the torque exerted between two arc-shaped permanent magnets uses a very simple expression which is in the form $\left(-\frac{\eta}{a b} \arctan \left[\frac{\xi}{\eta}\right]+\frac{\xi}{a b}\right)$. This is interesting for different points of view. First, these expressions are rather simple to compute and, according to the precision required in the calculation of the azimuthal force or the torque, this very simple expression keeps the same form. Second, they have a very low computational cost.

More generally, all the results obtained in this paper can be very useful for scientifics involved in the design of synchronous couplings. Indeed, they are a good alternative to the finite element method which requires often a high computational cost.

\section{EXPRESSION OF THE AZIMUTHAL FIELD CREATED BY ONE ARC-SHAPED PERMANENT MAGNET RADIALLY MAGNETIZED}

This section presents the expression of the azimuthal field created by one arc-shaped permanent magnet whose polarization is radial. It is noted that is expression differs from the one determined in a previous paper [31] because the magnetic charge volume density is taken into account in this paper. Consequently, this expression can be easily used for studying the effects of the magnetic field created by one magnet on the demagnetizing field of another magnet.

\section{A. Notation and geometry}

The geometry considered and the related parameters are shown in Fig 1. The arc-shaped inner radius is $r_{i n 1}$ and its outer one is $r_{\text {out } 1}$. Its height is $h$. The axis $z$ is an axis of symmetry. Calculations are obtained by using the Coulombian model. Consequently, the arc-shaped permanent magnet is represented by two arc-shaped planes which correspond to the inner and outer faces of the ring and an arc-shaped volume inside the magnet. The inner arc-shaped plane is charged with a magnetic pole surface density $+\sigma_{s}^{*}$ and the lower one is charged with the opposite magnetic pole surface density $-\sigma_{s}^{*}$. The arc-shaped volume inside the magnet is charged with the magnetic pole volume density $\sigma_{v}^{*}$.

The charge densities must verify (1).

$$
\iint_{S_{\text {in }}} \sigma_{s}^{*} d s_{\text {in }}-\iint_{S_{\text {out }}} \sigma_{s}^{*} d s_{\text {out }}+\iiint_{V} \sigma_{v}^{*} d v=0
$$

where $S_{\text {in }}$ and $S_{\text {out }}$ denote the inner and outer arc-shaped surfaces of the magnet and $V$ is its volume. When the width of the magnet is very small, the expression of the azimuthal field has been determined in a previous paper [31]. Consequently, we only explain here how to find the magnetic field created by the magnetic charge volume density. Such a case corresponds to thick or thin arc-shaped permanent 


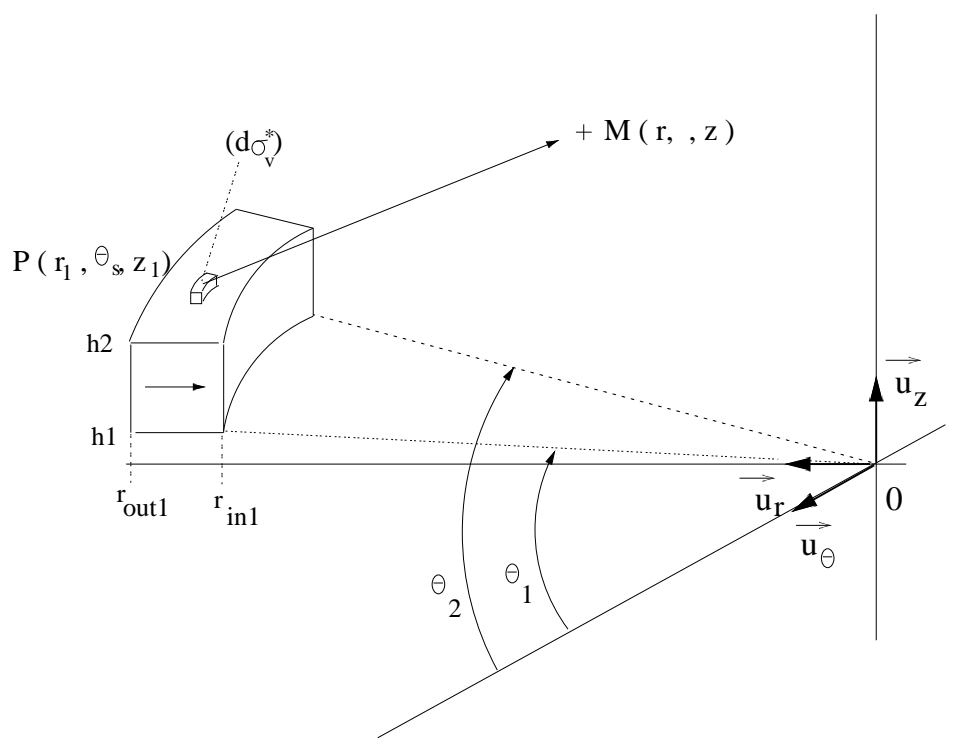

Fig. 1. Representation of the configuration considered. The ring inner radius is $r_{i n_{1}}$, the ring outer one is $r_{o u t_{1}}$, its height is $h$, its angular width is $\theta_{2}-\theta_{1}$

magnet rings. It is noted that the total azimuthal field created by this arc-shaped permanent magnet can be determined by using the principle of superposition. Indeed, this azimuthal field is the sum of all the charge contributions of the magnet.

\section{B. Basic Equation}

We explain now how to determine the azimuthal field created by the magnetic pole volume density of the arc-shaped permanent magnet shown in Fig. 1. The expression obtained uses nor special functions neither elliptic integrals. As a consequence, we can say that this expression is fully analytical.

Let us consider a point $P\left(r_{1}, \theta_{s}, z_{1}\right)$ which is located in the elementary volume of the ring and an observation point $M(r, \theta, z)$. The azimuthal component of the magnetic field created by this elementary volume can be determined by calculating the projection of the vector $\overrightarrow{P M}$ along $\vec{u}_{\theta}$.

$$
H_{\theta}(r, \theta, z)=\frac{1}{4 \pi \mu_{0}} \int_{r_{i_{1}}}^{r_{\text {out }_{1}}} \int_{\theta_{1}}^{\theta_{2}} \int_{0}^{h} \frac{\left(\overrightarrow{P M} \cdot \vec{u}_{\theta}\right)}{|\overrightarrow{P M}|^{3}} d \sigma_{v}^{*}
$$

where $d \sigma_{v}^{*}$ is the elementary volume charged with the magnetic pole volume density.

$$
d \sigma_{v}^{*}=\sigma_{v}^{*} r_{1} d r_{1} d \theta_{s} d z_{1}
$$

where $\sigma_{v}^{*}$ is the magnetic pole volume density.

In cyclindrical coordinates, the magnetic pole volume density $\sigma_{v}^{*}$ can be written as follows

$$
\sigma_{v}^{*}=\frac{J}{r_{1}}
$$


Moreover, we have

$$
\overrightarrow{P M} \cdot \vec{u}_{\theta}=-r_{1} \sin \left[\theta-\theta_{s}\right]
$$

and

$$
|\overrightarrow{P M}|^{3}=\left(r^{2}+r_{1}^{2}-2 r r_{1} \cos \left[\theta-\theta_{s}\right]+\left(z-z_{1}\right)^{2}\right)^{\frac{3}{2}}
$$

We can now evaluate (2). With (3), (4), (5) and (6), (2) can be simplified.

$$
H_{\theta}(r, \theta, z)=\frac{J}{4 \pi \mu_{0}} \int_{r_{\text {in }_{1}}}^{r_{\text {out }}} \int_{\theta_{1}}^{\theta_{2}} \int_{0}^{h} \frac{-r_{1} \sin \left[\theta-\theta_{s}\right]}{\left(r^{2}+r_{1}^{2}-2 r r_{1} \cos \left[\theta-\theta_{s}\right]+\left(z-z_{1}\right)^{2}\right)^{\frac{3}{2}}} d r_{1} d \theta_{1} d z_{1}
$$

C. Determination of the azimuthal field created by the magnetic pole volume density

As the expression of the azimuthal field is entirely analytical, that is, without special functions (elliptic integrals, lambda function,...), this section presents the way of obtaining such an expression.

The first integration is done with respect to $\theta_{s}$. Indeed, we can use (8) in order to evaluate this first integration.

$$
\int_{\theta_{s}} \frac{\sin \left[\theta-\theta_{s}\right]}{\left(a+b \cos \left[\theta-\theta_{s}\right]\right)^{\frac{3}{2}}} d \theta_{s}=\frac{-2}{b \sqrt{a+b \cos \left[\theta-\theta_{s}\right]}}
$$

By using (8), we deduct that (7) becomes (9).

$$
H_{\theta}(r, \theta, z)=\frac{J}{4 \pi \mu_{0}} \int_{r_{i_{1}}}^{r_{\text {out }_{1}}} \int_{0}^{h} \alpha_{r_{1}, z_{1}} d r_{1} d z_{1}
$$

with

$$
\alpha_{r_{1}, z_{1}}=\frac{-1}{r \sqrt{r^{2}+r_{1}^{2}+\left(z-z_{1}\right)^{2}-2 r r_{1}} \cos \left[\theta-\theta_{1}\right]}+\frac{1}{r \sqrt{r^{2}+r_{1}^{2}+\left(z-z_{1}\right)^{2}-2 r r_{1} \cos \left[\theta-\theta_{2}\right]}}
$$

The following integration can be done with respect to $r_{1}$. We see that $\alpha_{r_{1}, z_{1}}$ has two terms which are equivalent when integrated according to $r_{1}$. Consequently, in order to simplify the notations, we will only consider one term of $\alpha_{r_{1}, z_{1}}$ and the second one can be integrated in the same way. The final result $H_{\text {theta }}$ is presented in (15). So we only explain here how to obtain the two last integrations. The integration according to $r_{1}$ can be done by using (11).

$$
\int_{r_{1}} \frac{1}{\sqrt{c+r_{1}^{2}-2 r_{1} d}} d r_{1}=\log \left[-d+r_{1}+\sqrt{e-2 d r_{1}+r_{1}^{2}}\right]
$$

By applying (11) to (9) and by considering only one term of $\alpha_{r_{1}, z_{1}}$, we find:

$$
H_{\theta}(r, \theta, z)=\frac{J}{4 \pi \mu_{0}} \int_{0}^{h} \beta_{z_{1}} d z_{1}
$$


with

$$
\begin{aligned}
& \beta\left(z_{1}\right)=\frac{1}{r}\left(\log \left[r_{i n_{1}}-r \cos \left[\theta-\theta_{1}\right]+\sqrt{r^{2}+r_{i n_{1}}^{2}+\left(z-z_{1}\right)^{2}-2 r r_{i n_{1}} \cos \left[\theta-\theta_{2}\right]}\right]\right) \\
& -\frac{1}{r}\left(\log \left[r_{i n_{1}}-r \cos \left[\theta-\theta_{2}\right]+\sqrt{r^{2}+r_{i n_{1}}^{2}+\left(z-z_{1}\right)^{2}-2 r r_{i n_{1}} \cos \left[\theta-\theta_{2}\right]}\right]\right) \\
& -\frac{1}{r}\left(\log \left[r_{\text {out }_{1}}-r \cos \left[\theta-\theta_{1}\right]+\sqrt{r^{2}+r_{\text {out }_{1}}^{2}+\left(z-z_{1}\right)^{2}-2 r r_{\text {out }_{1}} \cos \left[\theta-\theta_{1}\right]}\right]\right)
\end{aligned}
$$

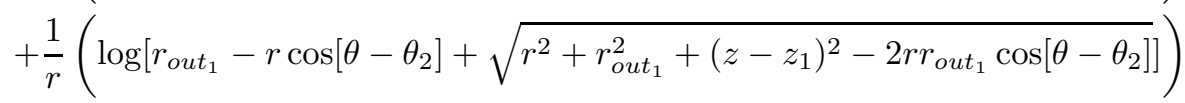

We see that $\beta\left(z_{1}\right)$ has four terms which are equivalent when integrated according to $z_{1}$. Consequently, we only consider one term and we use the relation (14) to integrate it according to $z_{1}$.

$$
\begin{aligned}
\int_{z_{1}} \log \left[e+\sqrt{f+\left(z-z_{1}\right)^{2}}\right] d z_{1}= & -z_{1}-\sqrt{-e^{2}+f} \arctan \left[\frac{z-z_{1}}{\sqrt{-e^{2}+f}}\right] \\
& -\frac{\left(e^{2}-f\right) \arctan \left[\frac{e\left(z-z_{1}\right)}{\sqrt{-e^{2}+f} \sqrt{f+\left(z-z_{1}\right)^{2}}}\right]}{\sqrt{-e^{2}+f}} \\
& -\left(z-z_{1}\right) \log \left[e+\sqrt{f+\left(z-z_{1}\right)^{2}}\right] \\
& -e \log \left[z+\sqrt{f+\left(z-z_{1}\right)^{2}}-z_{1}\right]
\end{aligned}
$$

By applying (14) to (12) and by taking into account all the terms omitted previously, we obtain (15).

$$
H_{\theta}=\frac{J}{4 \pi \mu_{0}} e\left(r_{\text {in }_{1}}, r_{\text {out }}, 0, h, r, z, \theta, \theta_{1}, \theta_{2}\right)
$$

with

$$
e\left(r_{\text {in }_{1}}, r_{\text {out }}, 0, h, r, z, \theta, \theta_{1}, \theta_{2}\right)=g\left(r_{\text {in }_{1}}, r_{\text {out }}, h, r, z, \theta, \theta_{1}, \theta_{2}\right)-g\left(r_{\text {in }_{1}}, r_{\text {out }},{ }_{1}, 0, r, z, \theta, \theta_{1}, \theta_{2}\right)
$$

and

$$
\begin{aligned}
g\left(r_{\text {in }_{1}}, r_{\text {out }_{1}}, z_{1}, r, z, \theta, \theta_{1}, \theta_{2}\right) & =h\left(r_{\text {out }}, z_{1}, r, z, \theta, \theta_{1}, \theta_{2}\right)-h\left(r_{\text {in }}, z_{1}, r, z, \theta, \theta_{1}, \theta_{2}\right) \\
h\left(r_{1}, z_{1}, r, z, \theta, \theta_{1}, \theta_{2}\right) & =f\left(r_{1}, z_{1}, r, z, \theta, \theta_{2}\right)-f\left(r_{1}, z_{1}, r, z, \theta, \theta_{1}\right) \\
f\left(r_{1}, z_{1}, r, z, \theta, \theta_{s}\right)= & \frac{1}{r}\left(-z_{1}-\left(r_{1}-r \cos \left[\theta-\theta_{s}\right]\right) \log \left[z-z_{1}+\eta\right]\right) \\
& +\frac{1}{r}\left(\left(z-z_{1}\right) \log \left[r_{1}-r \cos \left[\theta-\theta_{s}\right]+\eta\right]\right) \\
& +\arctan \left[\frac{r \sin \left[\theta-\theta_{s}\right]}{z-z_{1}}\right] \sin \left[\theta-\theta_{s}\right] \\
& +\sin \left[\theta-\theta_{s}\right] \arctan \left[\frac{\left(z-z_{1}\right)\left(r-r_{1} \cos \left[\theta-\theta_{s}\right]\right)}{r \sin \left[\theta-\theta_{s}\right] \eta}\right]
\end{aligned}
$$




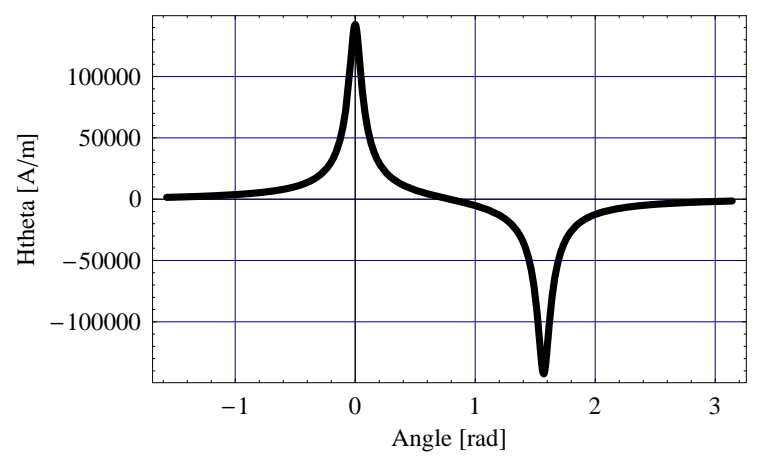

Fig. 2. Representation of the azimuthal field versus $\theta$. We take $J=1 T, r_{\text {in }_{1}}=0.025, r_{\text {out }_{1}}=0.028, h=0.003, \theta_{2}-\theta_{1}=\frac{\pi}{2}$

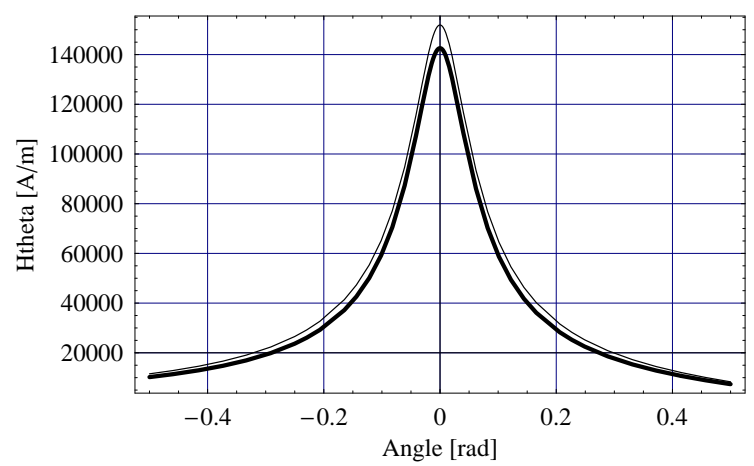

Fig. 3. Representation of the azimuthal field versus $\theta$. We take $J=1 T, r_{\text {in }_{1}}=0.025, r_{o u t}=0.028, h=0.003, \theta_{2}-\theta_{1}=\frac{\pi}{2}$ (thick line = with $\sigma_{v}^{*}$, thin line $=$ without $\sigma_{v}^{*}$ )

with

$$
\eta=\sqrt{r^{2}+r_{1}^{2}+\left(z-z_{1}\right)^{2}-2 r r_{1} \cos \left[\theta-\theta_{s}\right]}
$$

We can conclude that a fully analytical expression of the azimuthal field exists for arc-shaped permanent magnets whose polarization is radial. This result is of great importance because the evaluation of this azimuthal field is exact and has a very low computational cost. Fig. 2 represents the total azimuthal field created by the arc-shaped permanent magnet shown in Fig. 1.

\section{Interest of taking into account the magnetic pole volume density}

We have shown that it is possible to obtain a fully analytical expression of the azimuthal field created by one arc-shaped permanent magnet. However, studies dealing with magnetic couplings often require expressions of the magnetic forces exerted between arc-shaped permanent magnets. The calculation of such forces is rather difficult in the case of arc-shaped permanent magnets radially magnetized because many integrations must be determined. Thereby, it can be interesting to know if the magnetic pole volume density must be taken into account for determining the azimuthal field. Fig. 3 represents this azimuthal field with or without the magnetic pole volume density. We see in Fig. 3 that a slight error is done in the 
evaluation of the azimuthal field when the magnetic charge volume density is omitted. For example, an exact calculation shows that this error equals $6.1 \%$ in Fig. 2 when $\theta=0^{\circ}$.

It can be concluded that the magnetic pole volume density must be taken into account for calculating the azimuthal field created by arc-shaped permanent magnets whose polarization is radial. Consequently, this magnetic pole volume density must be taken into account for calculating the azimuthal force exerted between two arc-shaped permanent magnets. Such a calculation is presented in next section.

\section{SEMI-ANALYTICAL EXPRESSION OF THE AZIMUTHAL FORCE CREATED BETWEen TWO ARC-SHAPED PERMANENT MAGNETS}

This section presents a semi-analytical expression of the azimuthal force exerted between two arc-shaped permanent magnet rings whose polarization is radial.

\section{A. Notation and geometry}

The geometry considered and the related parameters are shown in Fig 4. For the left arc-shaped permanent magnet, the inner radius is $r_{i n 1}$ and its outer one is $r_{\text {out } 1}$. Its height is $h=h 2-h 1$ and its angular width is $\theta_{2}-\theta_{1}$. For the right arc-shaped permanent magnet, the inner radius is $r_{i n 2}$ and its outer one is $r_{\text {out } 2}$. Its height is $z b-z a$ and its angular width is $\theta_{4}-\theta_{3}$. The axis $z$ is an axis of symmetry. Calculations are obtained by using the Coulombian model. Consequently, each arc-shaped permanent magnet is represented by two arc-shaped planes which correspond to the inner and outer faces of the ring and an arc-shaped volume inside the magnet. For each case, the inner arc-shaped plane is charged with a magnetic pole surface density $+\sigma_{s}^{*}$ and the lower one is charged with the opposite magnetic pole surface density $-\sigma_{s}^{*}$. The arc-shaped volume inside the magnet is charged with the magnetic pole volume density $\sigma_{v}^{*}$.

B. Expression of the azimuthal force exerted between two arc-shaped permanent magnets radially magnetized

The azimuthal force can be determined by integrating the azimuthal field created by the left arc-shaped permanent magnet on the charge distribution located on the right arc-shaped permanent magnet. There are three charge distributions for each arc-shaped permanent magnet, therefore, there are nine terms to determine. As we have shown in the previous section that the magnetic pole volume density has an influence on the magnetic field created, we must take into account all the terms which correspond to three types of interactions between the two arc-shaped permanent magnets. This leads to write the azimuthal force as follows:

$$
\begin{aligned}
F_{\theta}= & -\frac{\sigma_{1}^{*} \sigma_{2}^{*}}{4 \pi \mu_{0}} \int_{\theta_{1}}^{\theta_{2}} \int_{0}^{h} \int_{\theta_{3}}^{\theta_{4}} \int_{z_{a}}^{z_{b}} \frac{-r_{\text {in } 1}^{2} r_{\text {out } 2} \sin \left(\theta_{j}-\theta_{i}\right)}{r_{\text {out } 2}^{2}+r_{\text {in } 1}^{2}-2 r_{\text {in } 1} r_{\text {out } 2} \cos \left(\theta_{j}-\theta_{i}\right)+\left(z_{2}-z_{1}\right)} d \theta_{i} d z_{1} d \theta_{j} d z_{2} \\
& +\frac{\sigma_{1}^{*} \sigma_{2}^{*}}{4 \pi \mu_{0}} \int_{\theta_{1}}^{\theta_{2}} \int_{0}^{h} \int_{\theta_{3}}^{\theta_{4}} \int_{z_{a}}^{z_{b}} \frac{-r_{\text {out } 1}^{2} r_{\text {out } 2} \sin \left(\theta_{j}-\theta_{i}\right)}{r_{\text {out } 2}^{2}+r_{\text {out } 1}^{2}-2 r_{\text {out } 1} r_{\text {out } 2} \cos \left(\theta_{j}-\theta_{i}\right)+\left(z_{2}-z_{1}\right)} d z_{i} d \theta_{j} d z_{2} \\
& +\frac{\sigma_{1}^{*} \sigma_{2}^{*}}{4 \pi \mu_{0}} \int_{\theta_{1}}^{\theta_{2}} \int_{0}^{h} \int_{\theta_{3}}^{\theta_{4}} \int_{z_{a}}^{z_{b}} \frac{-r_{\text {in } 1}^{2} r_{\text {in } 2} \sin \left(\theta_{j}-\theta_{i}\right)}{r_{\text {out } 2}^{2}+r_{\text {in } 1}^{2}-2 r_{\text {in } 1} r_{\text {out } 2} \cos \left(\theta_{j}-\theta_{i}\right)+\left(z_{2}-z_{1}\right)} d \theta_{i} d z_{1} d \theta_{j} d z_{2} \\
& -\frac{\sigma_{1}^{*} \sigma_{2}^{*}}{4 \pi \mu_{0}} \int_{\theta_{1}}^{\theta_{2}} \int_{0}^{h} \int_{\theta_{3}}^{\theta_{4}} \int_{z_{a}}^{z_{b}} \frac{-r_{\text {out } 1}^{2} r_{\text {in } 2} \sin \left(\theta_{j}-\theta_{i}\right)}{r_{\text {in } 2}^{2}+r_{\text {out } 1}^{2}-2 r_{\text {out } 1} r_{\text {in } 2} \cos \left(\theta_{j}-\theta_{i}\right)+\left(z_{2}-z_{1}\right)} d z_{j} d z_{2} \\
& -\frac{\sigma_{1}^{*} \sigma_{2}^{*}}{4 \pi \mu_{0}} \int_{r_{\text {in } 1}}^{r_{\text {out } 1}} \int_{\theta_{1}}^{\theta_{2}} \int_{0}^{h} \int_{\theta_{3}}^{\theta_{4}} \int_{z_{a}}^{z_{b}} \frac{-r_{1} r_{\text {out } 2} \sin \left(\theta_{j}-\theta_{i}\right)}{r_{1}^{2}+r_{\text {out } 2}^{2}-2 r_{\text {out } 2} r_{1} \cos \left(\theta_{j}-\theta_{i}\right)+\left(z_{2}-z_{1}\right)} d r_{1} d \theta_{i} d z_{1} d \theta_{j} d z_{2}
\end{aligned}
$$




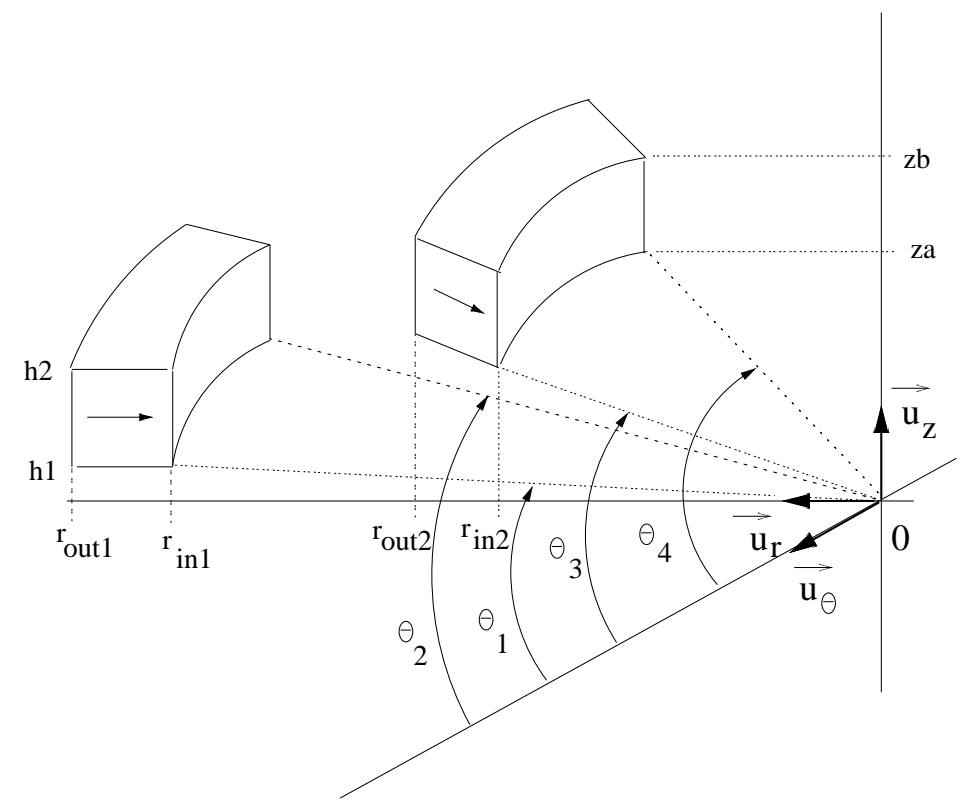

Fig. 4. Representation of the configuration considered. For the left arc-shaped permanent magnet, the ring inner radius is $r_{i n 1}$, the ring outer one is $r_{\text {out } 1}$, its height is $h=h 2-h 1$, its angular width is $\theta_{2}-\theta_{1}$; for the right arc-shaped permanent magnet, the ring inner radius is $r_{i n 2}$, the ring outer one is $r_{\text {out } 2}$, its height is $z b-z a$; its angular width is $\theta_{4}-\theta_{3}$

$$
\begin{aligned}
& +\frac{\sigma_{1}^{*} \sigma_{2}^{*}}{4 \pi \mu_{0}} \int_{r_{\text {in } 1}}^{r_{\text {out } 1}} \int_{\theta_{1}}^{\theta_{2}} \int_{0}^{h} \int_{\theta_{3}}^{\theta_{4}} \int_{z_{a}}^{z_{b}} \frac{-r_{1} r_{\text {in } 2} \sin \left(\theta_{j}-\theta_{i}\right)}{r_{1}^{2}+r_{\text {in } 2}^{2}-2 r_{\text {in } 2} r_{1} \cos \left(\theta_{j}-\theta_{i}\right)+\left(z_{2}-z_{1}\right)} d r_{1} d \theta_{i} d z_{1} d \theta_{j} d z_{2} \\
& -\frac{\sigma_{1}^{*} \sigma_{2}^{*}}{4 \pi \mu_{0}} \int_{r_{\text {in } 2}}^{r_{\text {out } 2}} \int_{\theta_{1}}^{\theta_{2}} \int_{0}^{h} \int_{\theta_{3}}^{\theta_{4}} \int_{z_{a}}^{z_{b}} \frac{-r_{2} r_{\text {out } 1} \sin \left(\theta_{j}-\theta_{i}\right)}{r_{2}^{2}+r_{\text {out } 1}^{2}-2 r_{2} r_{\text {out } 1} \cos \left(\theta_{j}-\theta_{i}\right)+\left(z_{2}-z_{1}\right)} d r_{2} d \theta_{i} d z_{1} d \theta_{j} d z_{2} \\
& +\frac{\sigma_{1}^{*} \sigma_{2}^{*}}{4 \pi \mu_{0}} \int_{r_{\text {in } 2}}^{r_{\text {out } 2}} \int_{\theta_{1}}^{\theta_{2}} \int_{0}^{h} \int_{\theta_{3}}^{\theta_{4}} \int_{z_{a}}^{z_{b}} \frac{-r_{2} r_{\text {in } 1} \sin \left(\theta_{j}-\theta_{i}\right)}{r_{2}^{2}+r_{\text {in } 1}^{2}-2 r_{2} r_{\text {in } 1} \cos \left(\theta_{j}-\theta_{i}\right)+\left(z_{2}-z_{1}\right)} d \theta_{i} d z_{1} d \theta_{j} d z_{2} \\
& +\frac{\sigma_{1}^{*} \sigma_{2}^{*}}{4 \pi \mu_{0}} \int_{r_{\text {in } 1}}^{r_{\text {out } 1}} \int_{r_{\text {in } 2}}^{r_{\text {out } 2}} \int_{\theta_{1}}^{\theta_{2}} \int_{0}^{h} \int_{\theta_{3}}^{\theta_{4}} \int_{z_{a}}^{z_{b}} \frac{-r_{1} \sin \left(\theta_{j}-\theta_{i}\right)}{r_{1}^{2}+r_{2}^{2}-2 r_{2} r_{1} \cos \left(\theta_{j}-\theta_{i}\right)+\left(z_{2}-z_{1}\right)} d r_{1} d r_{2} d \theta_{i} d z_{1} d \theta_{j} d z_{2}
\end{aligned}
$$

We see in Eq. (21) that there are three different integrands to integrate. These three different integrands correspond to the three types of interactions between the two arc-shaped permanent magnets. Indeed, we must determine the interaction between the magnetic charge surface densities, the interaction between the magnetic charge surface densities of one magnet and the magnetic charge volume density of the other one and the interaction between each magnetic charge volume density of the two magnets.

After having integrating according to $z_{1}, z_{2}$ and $\theta_{j}$, it rests a semi-analytical expression expressed as follows:

$$
F_{\theta}=\int_{\theta_{3}}^{\theta_{4}}\left(K_{1}^{\left(r_{\text {out } 1}, r_{\text {out } 2}\right)}-K_{1}^{\left(r_{\text {in } 1}, r_{\text {out } 2)}\right)}+K_{1}^{\left(r_{i n 1}, r_{i n 2}\right)}+K_{1}^{\left(r_{\text {out } 1}, r_{i n 2}\right)}\right) d \theta_{i}
$$




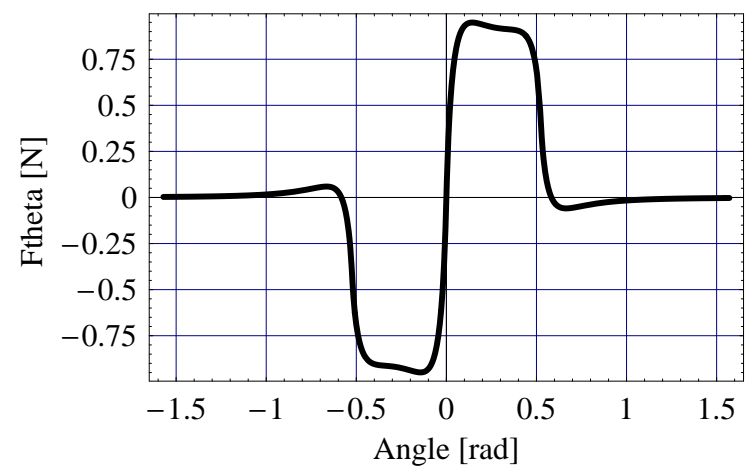

Fig. 5. Representation of the azimuthal force $f_{\theta}$ versus $\theta ; r_{\text {in } 1}=0.025 \mathrm{~m}, r_{\text {out } 1}=0.028 \mathrm{~m}, r_{\text {in } 2}=0.0219 \mathrm{~m}, r_{\text {out } 2}=0.0249 \mathrm{~m}$, $h 2-h 1=0.003 \mathrm{~m}, z b-z a=0.003 \mathrm{~m}, J=1 \mathrm{~T}, \theta_{2}-\theta_{1}=\theta_{4}-\theta_{3}=\frac{\pi}{4} \mathrm{rad}$.

$$
\begin{aligned}
& +\int_{\theta_{3}}^{\theta_{4}} \int_{r_{\text {in } 1}}^{r_{\text {out } 1}}\left(K_{2}^{\left(r_{1}, r_{\text {in } 2}\right)}-K_{2}^{\left(r_{1}, r_{\text {out } 2)}\right)}\right) d r_{1} d \theta_{i} \\
& +\int_{\theta_{3}}^{\theta_{4}} \int_{r_{\text {in } 2}}^{r_{\text {out } 2}}\left(K_{2}^{\left(r_{2}, r_{\text {in } 1}\right)}-K_{2}^{\left(r_{2}, r_{\text {out } 1}\right)}\right) d r_{2} d \theta_{i} \\
& +\int_{\theta_{3}}^{\theta_{4}} \int_{r_{\text {in } 1}}^{r_{\text {out } 1}} \int_{r_{\text {in } 2}}^{r_{\text {out } 2}} K_{3}^{\left(r_{1}, r_{2}\right)} d \theta_{i} d r_{1} d r_{2}
\end{aligned}
$$

with

$$
\begin{gathered}
K_{1}^{(a, b)}=-\frac{a^{2} b \sigma_{1}^{*} \sigma_{2}^{*}}{4 \pi \mu_{0}}\left(g\left(\theta_{2}\right)-g\left(\theta_{1}\right)\right) \\
K_{2}^{(a, b)}=-\frac{a b \sigma_{1}^{*} \sigma_{2}^{*}}{4 \pi \mu_{0}}\left(g\left(\theta_{2}\right)-g\left(\theta_{1}\right)\right) \\
K_{3}^{(a, b)}=-\frac{a \sigma_{1}^{*} \sigma_{2}^{*}}{4 \pi \mu_{0}}\left(g\left(\theta_{2}\right)-g\left(\theta_{1}\right)\right) \\
g\left(\theta_{j}\right)=\quad \beta\left(a, b, h 2, z a, \theta_{i}, \theta_{j}\right)-\beta\left(a, b, h 1, z a, \theta_{i}, \theta_{j}\right) \\
+\beta\left(a, b, h 1, z b, \theta_{i}, \theta_{j}\right)-\beta\left(a, b, h 2, z b, \theta_{i}, \theta_{j}\right) \\
\beta\left(a, b, z a, z b, a, b, \theta_{i}, \theta_{j}\right)=-\frac{\eta}{a b} \arctan \left[\frac{\xi}{\eta}\right]+\frac{\xi}{a b}
\end{gathered}
$$

with

$$
\begin{gathered}
\xi=\sqrt{a^{2}+b^{2}+\left(z_{2}-z_{1}\right)^{2}-2 a b \cos \left(\theta_{i}-\theta_{j}\right)} \\
\eta=\sqrt{-\left(z_{1}-z_{2}\right)^{2}}
\end{gathered}
$$

Fig. 5 represents the azimuthal force between the two arc-shaped permanent magnets versus the angle $\theta$. 


\begin{tabular}{|l|c|}
\hline terms & Computational cost of $H_{\theta}$ \\
\hline$\sigma_{s 1}^{*} \sigma_{s 2}^{*}$ & $0.016 \mathrm{~s}$ \\
\hline$\sigma_{s 1}^{*} \sigma_{s 2}^{*}+\sigma_{s 1}^{*} \sigma_{v 2}^{*}+\sigma_{s 2}^{*} \sigma_{v 1}^{*}$ & $0.0187 \mathrm{~s}$ \\
\hline$\sigma_{s 1}^{*} \sigma_{s 2}^{*}+\sigma_{s 1}^{*} \sigma_{v 2}^{*}+\sigma_{s 2}^{*} \sigma_{v 1}^{*}+\sigma_{v 1}^{*} \sigma_{v 2}^{*}$ & $23.12 \mathrm{~s}$ \\
\hline
\end{tabular}

TABLE I

COMPUTATIONAL COST OF THE AZIMUTHAL FIELD $H_{\theta}$ CREATED BY ONE ARC-SHAPED PERMANENT MAGNET RADIALLY MAGNETIZED.

\section{Possibility of omitting some terms in the expression of the azimuthal force}

We have presented a semi-analytical expression of the azimuthal force which is valid for thin or thick arc-shaped permanent magnets. This expression shows clearly that there three types of interactions between the two arc-shaped permanent magnets. An interesting point to notify is that this expression consists in integrating one, two or three times a very simple expression of the form $-\frac{\eta}{a b} \arctan \left[\frac{\xi}{\eta}\right]+\frac{\xi}{a b}$. This is interesting because according to the precision of the calculation required, this expression has always the same form; only the number of numerical integrations can be add or not.

Moreover, as the aim of such an expression is also to allow easy parametric optimizations of the arcshaped permanent magnets, it is very interesting to try to simplify Eq. (22). The major problem of Eq. (22) is the number of numerical integrations necessary to determine the azimuthal force. The more the number of numerical integrations is important, the more the computational cost is. We see in Eq. (22) that the term which describes the interaction between the two magnetic pole surface densities of each arc-shaped permanent magnet must be integrated numerically three times.

For example, we can compare the time necessary to determine the azimuthal field between two arcshaped permanent magnets for the following dimensions : $r_{i n 1}=0.025, r_{\text {out } 1}=0.028, r_{i n 2}=0.0219$, $r_{\text {out } 1}=0.0249, \theta_{2}-\theta_{1}=\frac{\pi}{6}, \theta_{4}-\theta_{3}=\frac{\pi}{6}$ with $\theta_{3}=\frac{\pi}{12}$. We present the computational cost values in table I. Let us precise that $\sigma_{s}^{*} \sigma_{s}^{*}$ corresponds to the case when we have only taken into account the magnetic pole surface densities of each arc-shaped permanent magnet. In addition, $\sigma_{s}^{*} \sigma_{v}^{*}$ corresponds to the interaction between the magnetic pole surface densities and the magnetic pole volume densities of each permanent magnet and $\sigma_{v}^{*} \sigma_{v}^{*}$ corresponds to the magnetic pole volume densities of each permanent magnet. Furthermore, in table $\mathrm{I}, \sigma_{s i}^{*}$ corresponds to the two surface densities of the magnet $i$ and $\sigma_{v i}^{*}$ corresponds to the magnetic pole volume density of the magnet $i$.

Table I shows clearly that the term describing the interaction between the magnetic charge volume density of each ring has a great influence on the time necessary to determine the azimuthal force. It could be interesting to know if this term can be omitted or not but after several simulations, it seems that we must taken into account the interaction between the magnetic charge volume density of each arc-shaped permanent magnet. As a consequence, for parametric studies, it can be rather simple to use only $\sigma_{s}^{*} \sigma_{s}^{*}$ and $\sigma_{s}^{*} \sigma_{v}^{*}$ because these interactions have a low computational cost. Once the parametric study is finished, it can be interesting to use the third interaction $\sigma_{v}^{*} \sigma_{v}^{*}$ in order to obtain a very precise value of the torque transmitted between two arc-shaped permanent magnets.

\section{SEMi-ANALYTICAL EXPRESSiON OF THE TORQUE EXERTED BETWEEN TWO ARC-SHAPED PERMANENT MAGNETS RADIALLY MAGNETIZED}

This section presents a semi-analytical expression of the Torque exerted between two arc-shaped permanent magnets whose polarization is radial. It is to be noted that parametric optimizations in permanent magnet couplings often require the calculation of the torque exerted by a rotor on another one. 
The way of obtaining the torque between two arc-shaped permanent magnets radially magnetized is also based on the coulombian model. By using Fig. 4, the torque can be determined by integrating the magnetic field azimuthal component created by the left outer arc-shaped permanent magnets on the charge densities of the inner arc-shaped permanent magnet times the elementary distance $r$ which can be merely $r_{\text {in } 2}, r_{\text {out } 2}$ or $r_{2}$. Thus, this calculation is in fact quite similar to the previous one. However, we must take into account the elementary distance in the path of integration for calculation the torque transmitted by the outer arc-shaped permanent magnet on the inner one.

Thereby, the torque exerted by the outer arc-shaped permanent magnet on the inner one can be determined as follows:

$$
\begin{aligned}
& T_{\theta}=-\frac{\sigma_{1}^{*} \sigma_{2}^{*}}{4 \pi \mu_{0}} \int_{\theta_{1}}^{\theta_{2}} \int_{0}^{h} \int_{\theta_{3}}^{\theta_{4}} \int_{z_{a}}^{z_{b}} \frac{-r_{\text {in } 1}^{2} r_{\text {out } 2}^{2} \sin \left(\theta_{j}-\theta_{i}\right)}{r_{\text {out } 2}^{2}+r_{\text {in } 1}^{2}-2 r_{\text {in } 1} r_{\text {out } 2} \cos \left(\theta_{j}-\theta_{i}\right)+\left(z_{2}-z_{1}\right)} d \theta_{i} d z_{1} d \theta_{j} d z_{2} \\
& +\frac{\sigma_{1}^{*} \sigma_{2}^{*}}{4 \pi \mu_{0}} \int_{\theta_{1}}^{\theta_{2}} \int_{0}^{h} \int_{\theta_{3}}^{\theta_{4}} \int_{z_{a}}^{z_{b}} \frac{-r_{\text {out } 1}^{2} r_{\text {out } 2}^{2} \sin \left(\theta_{j}-\theta_{i}\right)}{r_{\text {out } 2}^{2}+r_{\text {out } 1}^{2}-2 r_{\text {out } 1} r_{\text {out } 2} \cos \left(\theta_{j}-\theta_{i}\right)+\left(z_{2}-z_{1}\right)} d \theta_{i} d z_{1} d \theta_{j} d z_{2} \\
& +\frac{\sigma_{1}^{*} \sigma_{2}^{*}}{4 \pi \mu_{0}} \int_{\theta_{1}}^{\theta_{2}} \int_{0}^{h} \int_{\theta_{3}}^{\theta_{4}} \int_{z_{a}}^{z_{b}} \frac{-r_{\text {in } 1}^{2} r_{\text {in } 2}^{2} \sin \left(\theta_{j}-\theta_{i}\right)}{r_{\text {out } 2}^{2}+r_{\text {in } 1}^{2}-2 r_{\text {in } 1} r_{\text {out } 2} \cos \left(\theta_{j}-\theta_{i}\right)+\left(z_{2}-z_{1}\right)} d \theta_{i} d z_{1} d \theta_{j} d z_{2} \\
& -\frac{\sigma_{1}^{*} \sigma_{2}^{*}}{4 \pi \mu_{0}} \int_{\theta_{1}}^{\theta_{2}} \int_{0}^{h} \int_{\theta_{3}}^{\theta_{4}} \int_{z_{a}}^{z_{b}} \frac{-r_{\text {out } 1}^{2} r_{\text {in } 2}^{2} \sin \left(\theta_{j}-\theta_{i}\right)}{r_{\text {in } 2}^{2}+r_{\text {out } 1}^{2}-2 r_{\text {out } 1} r_{\text {in } 2} \cos \left(\theta_{j}-\theta_{i}\right)+\left(z_{2}-z_{1}\right)} d \theta_{i} d z_{1} d \theta_{j} d z_{2} \\
& -\frac{\sigma_{1}^{*} \sigma_{2}^{*}}{4 \pi \mu_{0}} \int_{r_{\text {in } 1}}^{r_{\text {out } 1}} \int_{\theta_{1}}^{\theta_{2}} \int_{0}^{h} \int_{\theta_{3}}^{\theta_{4}} \int_{z_{a}}^{z_{b}} \frac{-r_{1} r_{\text {out } 2}^{2} \sin \left(\theta_{j}-\theta_{i}\right)}{r_{1}^{2}+r_{\text {out } 2}^{2}-2 r_{\text {out } 2} r_{1} \cos \left(\theta_{j}-\theta_{i}\right)+\left(z_{2}-z_{1}\right)} d r_{1} d \theta_{i} d z_{1} d \theta_{j} d z_{2} \\
& +\frac{\sigma_{1}^{*} \sigma_{2}^{*}}{4 \pi \mu_{0}} \int_{r_{\text {in } 1}}^{r_{\text {out } 1}} \int_{\theta_{1}}^{\theta_{2}} \int_{0}^{h} \int_{\theta_{3}}^{\theta_{4}} \int_{z_{a}}^{z_{b}} \frac{-r_{1} r_{i n 2}^{2} \sin \left(\theta_{j}-\theta_{i}\right)}{r_{1}^{2}+r_{i n 2}^{2}-2 r_{i n 2} r_{1} \cos \left(\theta_{j}-\theta_{i}\right)+\left(z_{2}-z_{1}\right)} d r_{1} d \theta_{i} d z_{1} d \theta_{j} d z_{2} \\
& -\frac{\sigma_{1}^{*} \sigma_{2}^{*}}{4 \pi \mu_{0}} \int_{r_{\text {in } 2}}^{r_{\text {out } 2}} \int_{\theta_{1}}^{\theta_{2}} \int_{0}^{h} \int_{\theta_{3}}^{\theta_{4}} \int_{z_{a}}^{z_{b}} \frac{-r_{2}^{2} r_{\text {out } 1} \sin \left(\theta_{j}-\theta_{i}\right)}{r_{2}^{2}+r_{\text {out } 1}^{2}-2 r_{2} r_{\text {out } 1} \cos \left(\theta_{j}-\theta_{i}\right)+\left(z_{2}-z_{1}\right)} d r_{2} d \theta_{i} d z_{1} d \theta_{j} d z_{2} \\
& +\frac{\sigma_{1}^{*} \sigma_{2}^{*}}{4 \pi \mu_{0}} \int_{r_{\text {in } 2}}^{r_{\text {out } 2}} \int_{\theta_{1}}^{\theta_{2}} \int_{0}^{h} \int_{\theta_{3}}^{\theta_{4}} \int_{z_{a}}^{z_{b}} \frac{-r_{2}^{2} r_{i n 1} \sin \left(\theta_{j}-\theta_{i}\right)}{r_{2}^{2}+r_{i n 1}^{2}-2 r_{2} r_{i n 1} \cos \left(\theta_{j}-\theta_{i}\right)+\left(z_{2}-z_{1}\right)} d r_{2} d \theta_{i} d z_{1} d \theta_{j} d z_{2} \\
& +\frac{\sigma_{1}^{*} \sigma_{2}^{*}}{4 \pi \mu_{0}} \int_{r_{\text {in } 1}}^{r_{\text {out } 1}} \int_{r_{\text {in } 2}}^{r_{\text {out } 2}} \int_{\theta_{1}}^{\theta_{2}} \int_{0}^{h} \int_{\theta_{3}}^{\theta_{4}} \int_{z_{a}}^{z_{b}} \frac{-r_{1} r_{2} \sin \left(\theta_{j}-\theta_{i}\right)}{r_{1}^{2}+r_{2}^{2}-2 r_{2} r_{1} \cos \left(\theta_{j}-\theta_{i}\right)+\left(z_{2}-z_{1}\right)} d r_{1} d r_{2} d \theta_{i} d z_{1} d \theta_{j} d z_{2}
\end{aligned}
$$

We can re-write (30) in a useful form which requires a low computational cost.

$$
\begin{aligned}
T_{\theta}= & \int_{\theta_{3}}^{\theta_{4}}\left(M_{1}^{\left(r_{\text {out } 1}, r_{\text {out } 2}\right)}-M_{1}^{\left(r_{\text {in } 1}, r_{\text {out } 2)}\right)}+M_{1}^{\left(r_{\text {in } 1}, r_{\text {in } 2}\right)}+M_{1}^{\left(r_{\text {out } 1}, r_{\text {in } 2}\right)}\right) d \theta_{i} \\
& +\int_{\theta_{3}}^{\theta_{4}} \int_{r_{\text {in } 1}}^{r_{\text {out } 1}}\left(M_{2}^{\left(r_{1}, r_{\text {in } 2}\right)}-M_{2}^{\left(r_{1}, r_{\text {out } 2}\right)}\right) d r_{1} d \theta_{i} \\
& +\int_{\theta_{3}}^{\theta_{4}} \int_{r_{\text {in } 2}}^{r_{\text {out } 2}}\left(M_{3}^{\left(r_{2}, r_{\text {in } 1}\right)}-M_{3}^{\left(r_{2}, r_{\text {out } 1}\right)}\right) d r_{2} d \theta_{i} \\
& +\int_{\theta_{3}}^{\theta_{4}} \int_{r_{\text {in } 1}}^{r_{\text {out } 1}} \int_{r_{\text {in } 2}}^{r_{\text {out } 2}} M_{4}^{\left(r_{1}, r_{2}\right)} d \theta_{i} d r_{1} d r_{2}
\end{aligned}
$$




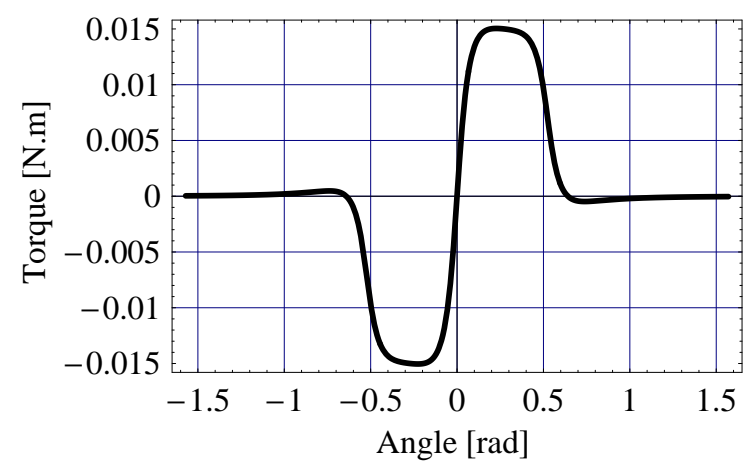

Fig. 6. Representation of the torque $T_{\theta}$ versus $\theta ; r_{\text {in } 1}=0.025 \mathrm{~m}, r_{\text {out } 1}=0.028 \mathrm{~m}, r_{\text {in } 2}=0.0219 \mathrm{~m}, r_{\text {out } 2}=0.0249 \mathrm{~m}$, $h 2-h 1=0.003 \mathrm{~m}, z b-z a=0.003 \mathrm{~m}, z a=0.001 \mathrm{~m}, J=1 \mathrm{~T}, \theta_{2}-\theta_{1}=\theta_{4}-\theta_{3}=\frac{\pi}{4} \mathrm{rad}$.

with

$$
\begin{aligned}
& M_{1}^{(a, b)}=-\frac{a^{2} b^{2} \sigma_{1}^{*} \sigma_{2}^{*}}{4 \pi \mu_{0}}\left(g\left(\theta_{2}\right)-g\left(\theta_{1}\right)\right) \\
& M_{2}^{(a, b)}=-\frac{a b^{2} \sigma_{1}^{*} \sigma_{2}^{*}}{4 \pi \mu_{0}}\left(g\left(\theta_{2}\right)-g\left(\theta_{1}\right)\right) \\
& M_{3}^{(a, b)}=-\frac{a^{2} B \sigma_{1}^{*} \sigma_{2}^{*}}{4 \pi \mu_{0}}\left(g\left(\theta_{2}\right)-g\left(\theta_{1}\right)\right) \\
& M_{4}^{(a, b)}=-\frac{a b \sigma_{1}^{*} \sigma_{2}^{*}}{4 \pi \mu_{0}}\left(g\left(\theta_{2}\right)-g\left(\theta_{1}\right)\right)
\end{aligned}
$$

and $g\left(\theta_{i}\right)$ is given by (26).

Fig. 6 illustrates this calculation with the following dimensions $: r_{i n 1}=0.025 \mathrm{~m}, r_{\text {out } 1}=0.028 \mathrm{~m}$, $r_{\text {in } 2}=0.0219 \mathrm{~m}, r_{\text {out } 2}=0.0249 \mathrm{~m}, h 2-h 1=0.003 \mathrm{~m}, z b-z a=0.003 \mathrm{~m}, z a=0.001 \mathrm{~m}, J=1 \mathrm{~T}$, $\theta_{2}-\theta_{1}=\theta_{4}-\theta_{3}=\frac{\pi}{4} \mathrm{rad}$.

Here again, for a simple parametric study, we could first take into account only the surface densities of each arc-shaped permanent magnet, that is by integrating only $M_{1}^{\left(r_{\text {out } 1}, r_{\text {out } 2}\right)}-M_{1}^{\left(r_{\text {in } 1}, r_{\text {out } 2}\right)}+$ $M_{1}^{\left(r_{i n 1}, r_{i n 2}\right)}+M_{1}^{\left(r_{o u t 1}, r_{i n 2}\right)}$ according to $\theta$. As there is only one numerical integration, this parametric study is very fast. After that, we can take into account the other contributions of Eq. (31) in order to obtain a more precise value of the torque or a more precision representation of this torque versus $\theta$.

In any case, such an approach allows to optimize easily the arc-shaped permanent magnet dimensions so as to obtain the torque required.

\section{CONCLUSION}

This paper has presented three important analytical relations which can be used for optimizing arcshaped permanent magnets radially magnetized in couplings. First, we have presented a fully analytical expression of the azimuthal field created by an arc-shaped permanent magnet. This expression uses neither special functions nor elliptic integrals. This is interesting because it means that a field created by a curved volume charge can be determined without using any approximations or numerical integrations. Then, 
this paper has presented a semi-analytical expression of the azimuthal force exerted between two arcshaped permanent magnets. Such an expression is very useful for optimizing for example the magnet dimensions in order to obtain a great force. In addition, this expression is three-dimensional, that is, the radius of curvature is taken into account in the expressions obtained. At least, this paper has presented a three-dimensional expression of the torque transmitted by one arc-shaped permanent magnet on another arc-shaped permanent magnet, both having a radial polarization. We can say that the final semi-analytical expressions determined in this paper are in the following form $\left(-\frac{\eta}{a b} \arctan \left[\frac{\xi}{\eta}\right]+\frac{\xi}{a b}\right)$ and some parameters vary in the two expressions. Thereby, even if the initial problem was rather difficult to solve (for the azimuthal force or the torque transmitted), we can express these semi-analytical expressions in a very useful form. As many papers dealing with magnetic couplings used two-dimensional analytical or semianalytical expressions for studying magnetic couplings, the three expressions determined in this paper can also be used because they are three-dimensional and they have a low computational cost.

The Mathematica files containing the analytical expressions presented in this paper are given on line [51].

\section{REFERENCES}

[1] J. P. Yonnet, "Passive magnetic bearings with permanent magnets," IEEE Trans. Magn., vol. 14, no. 5, pp. 803-805, 1978.

[2] J. P. Yonnet, "Permanent magnet bearings and couplings," IEEE Trans. Magn., vol. 17, no. 1, pp. 1169-1173, 1981.

[3] J. P. Yonnet, S. Hemmerlin, E. Rulliere, and G. Lemarquand, "Analytical calculation of permanent magnet couplings," IEEE Trans. Magn., vol. 29, no. 6, pp. 2932-2934, 1993.

[4] P. Elies and G. Lemarquand, "Analytical optimization of the torque of a permanent-magnet coaxial synchronous coupling," IEEE Trans. Magn., vol. 34, no. 4, pp. 2267-2273, 1998.

[5] P. Elies and G. Lemarquand, "Analytical study of radial stability of permanent magnet synchronous couplings," IEEE Trans. Magn., vol. 35, no. 4, pp. 2133-2136, 1999.

[6] J. Delamare, E. Rulliere, and J. P. Yonnet, "Classification and synthesis of permanent magnet bearing configurations," IEEE Trans. Magn., vol. 31, no. 6, pp. 4190-4192, 1995.

[7] W. Baran and M. Knorr, "Synchronous couplings with sm co5 magnets," pp. 140-151, 2nd Int. Workshop on Rare-Earth Cobalt Permanent Magnets and Their Applications, Dayton, Ohio, USA, 1976.

[8] P. Samanta and H. Hirani, "Magnetic bearing configurations: Theoretical and experimental studies," IEEE Trans. Magn., vol. 44, no. 2, pp. 292-300, 2008.

[9] S. C. Mukhopadhyay, J. Donaldson, G. Sengupta, S. Yamada, C. Chakraborty, and D. Kacprzak, "Fabrication of a repulsivetype magnetic bearing using a novel arrangement of permanent magnets for vertical-rotor suspension," IEEE Trans. Magn., vol. 39 , no. 5 , pp. 3220-3222, 2003

[10] R. Moser, J. Sandtner, and H. Bleuler, "Optimization of repulsive passive magnetic bearings," IEEE. Trans. Magn., vol. 42, no. 8, pp. 2038-2042, 2006.

[11] E. P. Furlani, "Formulas for the force and torque of axial couplings," IEEE Trans. Magn., vol. 29, no. 5, pp. $2295-2301,1993$.

[12] E. Furlani, S. Reznik, and A. Kroll, "A three-dimensonal field solution for radially polarized cylinders," IEEE Trans. Magn., vol. 31 , no. 1 , pp. $844-851,1995$

[13] E. Furlani, "Field analysis and optimization of ndfeb axial field permanent magnet motors," IEEE Trans. Magn., vol. 33, no. 5, pp. 3883-3885, 1997.

[14] B. Azzerboni, E. Cardelli, and A. Tellini, "Computation of the magnetic field in massive conductor systems," IEEE Trans. on Magn., vol. 25, no. 6, pp. 4462-4473, 1989.

[15] B. Azzerboni and E. Cardelli, "Magnetic field evaluation for disk conductors," IEEE Trans. Magn., vol. 29, no. 6, pp. 2419$2421,1993$.

[16] B. Azzerboni and G. Saraceno, "Three-dimensional calculation of the magnetic field created by current-carrying massive disks," IEEE Trans. Magn., vol. 34, no. 5, pp. 2601-2604, 1998.

[17] M. Abele and H. A. Leupold, "A general method for flux confinement in permanent magnet structure," J. Appl. Phys., vol. 64, no. 10 , pp. 5988-5990, 1988.

[18] M. Abele, J. Jensen, and H. Rusinek, "Generation of uniform high fields with magnetized wedges," IEEE Trans. Magn., vol. 33, no. 5, pp. 3874-3876, 1997.

[19] K. Halbach, "Design of permanent multiple magnets with oriented rec material," Nucl. Inst. Meth., vol. 169, pp. 1-10, 1980.

[20] J. Hilton and S. McMurry, "Halbach cylinders with improved field homogeneity and tailored gradient fields," IEEE Trans. Magn., vol. 43, no. 5, pp. 1898-1902, 2007.

[21] C. Blache and G. Lemarquand, "New structures for linear displacement sensor with hight magnetic field gradient," IEEE Trans. Magn., vol. 28, no. 5, pp. 2196-2198, 1992.

[22] S. Babic, C. Akyel, S. Salon, and S. Kincic, "New expressions for calculating the magnetic field created by radial current in massive disks," IEEE Trans. Magn., vol. 38, pp. 497-500, March 2002. 
[23] S. Babic, C. Akyel, S. Salon, and S. Kincic, "New expressions for calculating the magnetic field created by radial current in massive disks," IEEE Trans. Magn., vol. 38, no. 2, pp. 497-500, 2002.

[24] J. P. Selvaggi, S. Salon, O. M. Kwon, and M. V. K. Chari, "Calculating the external magnetic field from permanent magnets in permanent-magnet motors - an alternative method," IEEE Trans. Magn., vol. 40, no. 5, pp. 3278-3285, 2004.

[25] J. Selvaggi, S. Salon, O. M. Kwon, and M. Chari, "Computation of the three-dimensional magnetic field from solid permanentmagnet bipolar cylinders by employing toroidal harmonics," IEEE Trans. Magn., vol. 43, no. 10, pp. 3833-3839, 2007.

[26] O. M. Kwon, C. Surussavadee, M. Chari, S. Salon, and K. Vasubramaniam, "Analysis of the far field of permanent magnet motors and effects of geometric asymmetries and unbalance in magnet design," IEEE Trans. Magn., vol. 40, no. 3, pp. 435-442, 2004.

[27] H. L. Rakotoarison, J. P. Yonnet, and B. Delinchant, "Using coulombian approach for modeling scalar potential and magnetic field of a permanent magnet with radial polarization," IEEE Trans. Magn., vol. 43, no. 4, pp. 1261-1264, 2007.

[28] Y. Zhilichev, "Calculation of magnetic field of tubular permanent magnet assemblies in cylindrical bipolar coordinates," IEEE Trans. Magn., vol. 43, no. 7, pp. 3189-3195, 2007.

[29] E. Perigo, R. Faria, and C. Motta, "General expressions for the magnetic flux density produced by axially magnetized toroidal permanent magnets," IEEE Trans. Magn., vol. 43, no. 10, pp. 3826-3832, 2008.

[30] E. Durand, "Electrostatique," Masson Editeur, Paris, France, vol. 1, pp. 248-251, 1964.

[31] R. Ravaud, G. Lemarquand, V. Lemarquand, and C. Depollier, "Analytical calculation of the magnetic field created by permanent-magnet rings," IEEE Trans. Magn., vol. 44, no. 8, pp. 1982-1989, 2008.

[32] F. Bancel and G. Lemarquand, "Three-dimensional analytical optimization of permanent magnets alternated structure," IEEE Trans. Magn., vol. 34, no. 1, pp. 242-247, 1998.

[33] V. Lemarquand, J. F. Charpentier, and G. Lemarquand, "Nonsinusoidal torque of permanent-magnet couplings," IEEE Trans. Magn., vol. 35, no. 5, pp. 4200-4205, 1999.

[34] J. F. Charpentier and G. Lemarquand, "Calculation of ironless permanent magnet coupling using semi-numerical magnetic pole theory method," COMPEL, vol. 20, no. 1, pp. 72-89, 2001.

[35] J. F. Charpentier and G. Lemarquand, "Optimization of unconventional p.m. couplings," IEEE Trans. Magn., vol. 38, no. 2 , pp. 1093-1096, 2002.

[36] G. Akoun and J. P. Yonnet, "3d analytical calculation of the forces exerted between two cuboidal magnets," IEEE Trans. Magn., vol. 20, no. 5, pp. 1962-1964, 1984.

[37] J. Conway, "Inductance calculations for noncoaxial coils using bessel functions," IEEE Trans. Magn., vol. 43, no. 3, pp. 10231034, 2007.

[38] J. Conway, "Noncoaxial inductance calculations without the vector potential for axisymmetric coils and planar coils," IEEE Trans. Magn., vol. 44, no. 10, pp. 453-462, 2008.

[39] K. Kim, E. Levi, Z. Zabar, and L. Birenbaum, "Mutual inductance of noncoaxial circular coils with constant current density," IEEE Trans. Magn., vol. 33, no. 32, pp. 4303-4309, 1997.

[40] S. Babic and C. Akyel, "Improvement of the analytical calculation of the magnetic field produced by permanent magnet rings," Progress in Electromagnetic Research, vol. 5, pp. 71-82, 2008.

[41] L. Yong, Z. Jibin, and L. Yongping, "Optimum design of magnet shape in permanent-magnet synchronous motors," IEEE Trans. Magn., vol. 39, no. 11, pp. 3523-4205, 2003.

[42] O. Cuguat, J. Delamare, and G. Reyne, "Magnetic micro-actuators and systems (magmas)," IEEE Trans. Magn., vol. 39, no. 5, pp. 3607-3612, 2003.

[43] J. Wang, G. W. Jewell, and D. Howe, "Design optimisation and comparison of permanent magnet machines topologies," vol. 148, pp. 456-464, IEE.Proc.Elect.Power Appl., 2001.

[44] M. Aydin, Z. Zhu, T. Lipo, and D. Howe, "Minimization of cogging torque in axial-flux permanent-magnet machines: design concepts," IEEE Trans. Magn., vol. 43, no. 9, pp. 3614-3622, 2007.

[45] J. P. Yonnet, Rare-earth Iron Permanent Magnets, ch. Magnetomechanical devices. Oxford science publications, 1996.

[46] T. Ohji, C. Mukhopadhyay, M. Iwahara, and S. Yamada, "Performance of repulsive type magnetic bearing system under nonuniform magnetization of permanent magnet," IEEE Trans. Magn., vol. 36, no. 5, pp. 3696-3698, 2000.

[47] T. Ohji, S. Ichiyama, K. Amei, M. Sakui, and S. Yamada, "Conveyance test by oscillation and rotation to a permanent magnet repulsive-type conveyoer," IEEE Trans. Magn., vol. 40, no. 4, pp. 3057-3059, 2004.

[48] M. Berkouk, V. Lemarquand, and G. Lemarquand, "Analytical calculation of ironless loudspeaker motors," IEEE Trans. Magn., vol. 37, no. 2, pp. 1011-1014, 2001.

[49] G. Lemarquand, "Ironless loudspeakers," IEEE Trans. Magn., vol. 43, no. 8, pp. 3371-3374, 2007.

[50] A. A. Hussien, S. Yamada, M. Iwahara, T. Okada, and T. Ohji, "Application of the repulsive-type magnetic bearing for manufacturing micromass measurement balance equipment," IEEE Trans. Magn., vol. 41, no. 10, pp. 3802-3804, 2005.

[51] http://www.univ-lemans.fr/ glemar. 